\title{
COMPETENCIAS PROFESIONALES Y SU \\ DESARROLLO MEDIANTE APRENDIZAJE- SERVICIO EN LA UNIVERSIDAD: PERSPECTIVA DE LOS EMPLEADORES ${ }^{1}$
}

\author{
PROFESSIONAL COMPETENCES AND THEIR \\ DEVELOPMENT THROUGH SERVICE-LEARNING \\ AT UNIVERSITIES: THE EMPLOYERS' PERSPECTIVE
}

\author{
Carolina Ugarte ${ }^{2}$ \\ https://orcid.org/0000-0002-2922-1101 \\ María Arantzamendi ${ }^{3}$ \\ https:// orcid.org/0000-0003-2406-0668 \\ Concepción Naval Durán ${ }^{4}$ \\ https: / / orcid.org/0000-0002-5927-9398
}

Natalia Verea $^{5}$
https://orcid.org/0000-0003-3279-7571

Elena Arbués ${ }^{6}$

Recibido: marzo 29, 2021 - Aceptado: mayo 17, 2021

\section{RESUMEN}

Introducirse en el mundo laboral es costoso y uno de los primeros obstáculos es el déficit competencial que, en ocasiones, presentan los universitarios. Para subsanar el exceso de una teoría descontextualizada, el EEES (Espacio Europeo de Educación Superior) ha auspiciado un cambio metodológico que fomenta una docencia más práctica, ya que el aprendizaje más efectivo es activo y está conectado con la experiencia. De este modo, la enseñanza universitaria se enfoca, entre otros aspectos, en la consecución de resultados de aprendizaje en forma de competencias asociadas al mercado laboral. En este contexto se están ensayando en la universidad [española] metodologías más prácticas y aplicadas, entre ellas, el Aprendizaje-Servicio.

1 Este trabajo se realizó en el marco del proyecto «Aprendizaje-Servicio e Innovación en la Universidad. Un programa para la mejora del rendimiento académico y el capital social de los estudiantes. MINECO Plan Estatal (2013-2016) EDU201341687-R (BOE 1/08/2014)», en el que participaron varias de las autoras. Actualmente, la investigación continúa en el marco del proyecto «Aprendizaje-Servicio (APS) y empleabilidad de los estudiantes universitarios en España: competencias para la inserción laboral» (MINECO Plan Estatal (2017-2021) EDU2017-82629-R).

2 Doctora en Teoría e Historia de la Educación, Universidad de Navarra, España. Dos sexenios de investigación reconocidos por la Agencia Nacional de la Actividad Investigadora, España.cugarte@unav.es

3 Doctora en Enfermería, Florence Nightingale School of Nursing \& Midwifery. Londres, Inglaterra. Investigadora de ATLANTES en el Instituto Cultura y Sociedad, Navarra, España. Profesora colaboradora, Facultad de Enfermería, Universidad de Navarra, España. marantz@unav.es

4 Doctora en Educación, Facultad de Humanidades y Ciencias Sociales, Universidad de Navarra. Catedrática «Teoría y Métodos en Educación y Psicología», Facultad de Humanidades y Ciencias Sociales, Universidad de Navarra. Profesora y Decana de la Facultad de Educación y Psicología, Universidad de Navarra. cnaval@unav.es

5 Máster en la Universidad a Distancia de Madrid, España. Coordinadora Practicum Educación, Universidad de Navarra, España. nverea@unav.es

6 Doctora en Eduacición. Profesora y Vicedecana de la Facultad de Educación y Psicología, Universidad de Navarra, España. earbues@unav.es 
El objetivo de este trabajo es conocer si para los empleadores participantes en nuestro estudio, los universitarios que participan en actividades de Aprendizaje-Servicio desarrollan el perfil competencial que buscan, haciéndoles candidatos más atractivos en los procesos de selección, mejorando en consecuencia su empleabilidad. Para ello, se desarrolló una investigación cualitativa genérica. Se realizaron tres grupos focales en los que participaron por muestreo intencional trece empleadores de egresados de distintas facultades de la universidad, pertenecientes a distintos sectores. La guía temática incluía preguntas sobre las competencias más demandadas por los empleadores, el Aprendizaje-Servicio y su influencia en el perfil profesional. La transcripción textual fue analizada mediante análisis de contenido inductivo por dos investigadores. En el apartado de resultados y discusión se constató que, para los empleadores consultados, los alumnos que participan en actividades de Aprendizaje-Servicio en la universidad, desarrollan algunas de las competencias profesionales más demandadas en el mundo laboral, incidiendo positivamente en su empleabilidad.

Palabras clave: aprendizaje, desarrollo de las habilidades, empleo, estudiante universitario, servicio, universidad.

\begin{abstract}
Entering the labour force is onerous and one of the first obstacles is, on occasion, the competence shortcomings of university students. To correct the excess of a decontextualized theory, the EHEA (European Higher Education Area) has sponsored a methodological change that encourages more practical teaching, since the most effective learning is active and connected with experience. In this way, university teaching focuses, among other aspects, on achieving learning results in the form of competences associated with the labour market. In this context, more practical and applied methodologies are being tested at the university, including Service-Learning. The objective of this study is to find out whether, for the employers participating in our study, the university students who participate in these Service-Learning activities develop the competency profile they are looking for, making them more attractive candidates in the selection processes, consequently improving their employability.
\end{abstract}

Generic quantitative research has been carried out. A purposive sampling of the employers of graduates from different university faculties was adopted. Three focal groups were formed using a topic guide, which addressed: the professional competences most demanded by employers; the repercussion of the teaching focus on employability; the social involvement capacity of their employees; Service-Learning and its influence on professional profile. 13 employers from different fields participated. Audio clips of the focal groups were recorded and their transcription was analysed by two researchers using content analysis.

In the view of the employers consulted, students who participate in Service-Learning activities at the university develop some of the most in-demand professional competences, which positively affect their employability.

Keywords: Employment, Learning, Service, Skills Development, Universities, University Students. 


\section{INTRODUCCIÓN}

La Estrategia Europa 2020 pretende dotar a los ciudadanos de las aptitudes adecuadas para los puestos de trabajo de hoy y de mañana. Esta meta ha conllevado que la Comisión añada entre sus prioridades, mejorar y actualizar la capacitación profesional abogando por un cambio fundamental en la educación, con una mayor focalización en los conocimientos, las capacidades y las competencias que adquieren los estudiantes.

Esta situación, afecta a la institución universitaria que, para adecuarse a las demandas del mercado laboral, asume el reto de formar profesionales que cuenten con conocimientos diferenciales y con competencias profesionales. A la hora de definir el perfil profesional de una titulación y determinar cuáles competencias debe reunir quien obtenga dicha titulación, es preciso contar no solo con los grupos internos de interés (la sociedad académica), sino también con los grupos de interés externos al proceso de educación (los empleadores). Por ello, en los últimos años, han aparecido numerosas investigaciones que analizan la inserción laboral de los recién egresados y las actuales demandas del mercado de trabajo en relación con los conocimientos y las habilidades que ha de poseer un titulado superior (Cedefop et al., 2017; OECD, 2017).

En este contexto cabe plantearse, a continuación, cuáles son las competencias profesionales para la empleabilidad y cómo pueden desarrollarse en la universidad.

\section{MARCO TEÓRICO: UNIVERSIDAD, COMPETENCIAS Y EMPLEABILIDAD}

\subsection{Competencias profesionales para la empleabilidad}

En la actualidad se asume la existencia de una serie de competencias profesionales que mejoran las posibilidades de encontrar empleo y mantenerlo, por el impacto que tienen en el desempeño del trabajo (Blokker, 2019). Las habilidades de comunicación oral y escrita, la colaboración y la resolución de problemas son competencias muy demandadas por los empleadores (Ríos et al., 2020).

En las últimas décadas, varios autores han sido referentes al proponer diferentes definiciones y categorías de competencias para el rendimiento efectivo y la empleabilidad (Gay et al., 1996; Goleman et al., 2002; Woodruffe, 1993). Más recientemente, Boyatzis define las competencias profesionales como comportamientos observables relacionados pero diferentes, organizados alrededor de un constructo subyacente, denominado intención (Boyatzis, 2011).

En los últimos años, esta perspectiva se ha complementado con la teoría sobre competencias, denominada Inteligencia Emocional Organizacional, que presta especial atención al contexto de la organización (Giorgi y Majer, 2012). Con este término, los autores se refieren a las relaciones de reciprocidad establecidas entre las competencias relacionales de las personas y el sistema organizacional en el que se integran. Esta teoría parte de la premisa de que las personas inteligentes hacen inteligentes a las organizaciones, y también estas organizaciones hacen inteligentes a las personas (Giorgi, 2013). De este modo, amplían el campo de la inteligencia emocional, centrada en el individuo y prestan más atención al contexto (Morone et al., 2016).

El perfil competencial para la empleabilidad demandado en las organizaciones, se configura por diferentes competencias profesionales. En el contexto español, destaca el modelo 
de Cardona y García-Lombardía (2005). Se selecciona este modelo competencial porque se considera que abarca de modo amplio y representativo la realidad de desempeño profesional que existe en nuestros entornos laborales. Estos autores clasifican las competencias en tres tipos. Las competencias estratégicas o de negocio son aquellas necesarias para obtener buenos resultados económicos. Incluirían la visión de negocio, la visión de la organización, la orientación al cliente, la gestión de recursos, la negociación y el networking. Por otra parte, estarían las competencias intratégicas o interpersonales, requeridas para el desarrollo de las personas, y el incremento de su compromiso y confianza con la empresa. Se encuentran entre ellas la comunicación, la gestión de conflictos, el carisma, el empowerment o delegación, el coaching y el trabajo en equipo. Finalmente, las competencias de eficacia personal, son hábitos que facilitan una relación eficaz de la persona con su entorno. Existen competencias de eficacia personal externa e interna. Las competencias de eficacia personal externa serían la proactividad, compuesta por la iniciativa, el optimismo y la ambición; y la gestión personal, integrada por la gestión del tiempo, la gestión de la información y la gestión del estrés. Las competencias de eficacia personal interna se ven conformadas por las competencias de mejora personal, que son la autocrítica, el autoconocimiento y el aprendizaje; y las competencias de autogobierno, que son la toma de decisiones, el autocontrol, el equilibrio emocional y la integridad.

\subsection{Desarrollo de competencias profesionales en la universidad, mediante el Aprendizaje-Servicio}

La preparación para el trabajo profesional es un tema candente en el planteamiento actual sobre el sentido y la misión de la universidad. Para que este proceso de cambio sea una realidad, los profesores deberán asumir el nuevo modelo educativo afrontando los retos metodológicos necesarios, teniendo en cuenta que el aprendizaje más efectivo es activo y que debe conectarse con la experiencia (Verea et al., 2018).

Cuanto más conocimiento y destreza transfieran los estudiantes universitarios desde el aula a la comunidad, y viceversa, mejor preparados estarán para afrontar lo que les ha de venir en su vida profesional (Santos Rego, 2020).

En el compromiso por dar cabida a un aprendizaje más activo y para introducir el aprendizaje de competencias profesionales en la universidad, se están ensayando distintas metodologías como los ejercicios de discusión y deliberación, los proyectos de investigación y acción política, la clase invertida, el aprendizaje basado en problemas o el aprendizaje cooperativo. De entre todas ellas destacamos el Aprendizaje-Servicio, por ser una metodología educativa innovadora ampliamente contrastada (Hora et al., 2016; Miller et al., 2018).

El Aprendizaje-Servicio permite a los estudiantes aprender conocimientos ligados al currículo de un modo significativo y contextualizado, a través de una experiencia de servicio organizado y voluntario, con intención de prestar atención a las necesidades sociales (Humburg et al., 2013; Jagla y Tice, 2019; Puig, 2009).

En estas actividades se incrementa el aprendizaje, se hace más significativo, cobra sentido y se multiplica su potencial formador. También se desarrollan prácticas valiosas para la formación de una ciudadanía activa y participativa, y para la inserción en el mundo laboral (Miller et al., 2018). Independientemente del tipo de servicio que se preste, en todos los casos las actividades de Aprendizaje-Servicio han de integrarse en el currículo 
académico, vinculadas a los objetivos de una asignatura para dar respuesta a necesidades reales y concretas de la comunidad, y aportar a los alumnos la oportunidad de adoptar nuevas habilidades y conocimientos basados en situaciones de la vida real.

Atendiendo a las aportaciones de los autores citados, se puede afirmar que, a través de la acción comprometida y reflexiva que surge en los proyectos de Aprendizaje-Servicio, se optimizan tanto el aprendizaje y la consolidación de los contenidos determinados en los planes de estudios, como el desarrollo de competencias profesionales. Sin embargo, queda por profundizar la perspectiva de los empleadores; y este trabajo contribuye a abordar dicho aspecto. Por tanto, el objetivo general es conocer si para los empleadores participantes en nuestro estudio, los universitarios que participan en estas actividades de Aprendizaje-Servicio desarrollan el perfil competencial que buscan, haciéndoles candidatos más atractivos en los procesos de selección y mejorando en consecuencia su empleabilidad.

En definitiva, se busca indagar el grado de correspondencia que para los empleadores consultados existe entre las competencias profesionales -que demandan en los procesos de selección-y las competencias profesionales -que creen que desarrollan los universitarios que participan en Aprendizaje-Servicio.

\section{MÉTODO}

Se realizó una investigación cualitativa genérica, entendida como aquella que no se guía por un conjunto explícito o establecido de suposiciones filosóficas en forma de una de las metodologías cualitativas definidas (Sandelowski, 2010). Este enfoque es adecuado cuando se quieren explorar perspectivas, en este caso, la de los empleadores.

\subsection{Objetivos de investigación}

El objetivo de este trabajo es conocer si para los empleadores participantes en nuestro estudio, los universitarios que participan en actividades de Aprendizaje-Servicio desarrollan el perfil competencial que buscan, haciéndoles candidatos más atractivos en los procesos de selección y mejorando en consecuencia su empleabilidad.

Para responder a esta pregunta, se plantean dos objetivos específicos:

Objetivo específico 1: Conocer cuáles son las competencias profesionales más demandadas por los empleadores de nuestro entorno.

Objetivo específico 2: Conocer cuáles son las competencias profesionales que los empleadores de nuestro entorno creen (si es que lo creen) que los universitarios desarrollan mediante la metodología del Aprendizaje-Servicio. 


\subsection{Muestra de la investigación}

Los posibles participantes eran los empleadores de egresados de la Universidad de Navarra. Teniendo en cuenta que en todas sus facultades se realizan proyectos de Aprendizaje-Servicio $^{7}$, se realizó un muestreo intencional considerando aspectos clave que promoviesen la heterogeneidad de la muestra y variedad de perspectivas.

Se contactó con los directores de desarrollo de todas las facultades para identificar empresas de distinto tamaño y sector (servicios, sanidad, producción, educación) que habitualmente empleaban a sus egresados. Se identificaron un total de cuarenta y dos posibles participantes, que fueron contactados por teléfono y / o correo electrónico, para invitarlos a participar e informarlos del objetivo de estudio y de lo que conllevaba su participación, tratando así de asegurar que disponían del conocimiento requerido sobre Aprendizaje-Servicio. Aceptaron participar trece representantes (siete mujeres y seis hombres) de empresas locales, nacionales y multinacionales, relacionadas con cuatro de los cinco ámbitos de conocimiento (Ciencias de la Salud, Ciencias Experimentales, Ciencias Sociales y Jurídicas, y Arte y Humanidades). Cinco de los trece participantes habían estado implicados directamente en proyectos de Aprendizaje-Servicio, llevados a cabo en sus empresas, en colaboración con dos facultades de la Universidad de Navarra. Los trece participantes que conformaban la muestra se caracterizaban por el perfil profesional que se describe en la siguiente tabla.

Tabla 1. Características de los participantes

\begin{tabular}{|c|c|c|c|c|}
\hline \multicolumn{2}{|c|}{ Criterio } & Grupo 1 & Grupo 2 & Grupo 3 \\
\hline \multirow[t]{4}{*}{ Sector } & Servicios & $x$ & $x$ & $X$ \\
\hline & Salud & $x$ & & $x$ \\
\hline & Producción & & $x$ & \\
\hline & Educación & $x$ & $x$ & $x$ \\
\hline \multirow{4}{*}{ Perfil del candidato } & $\begin{array}{l}\text { Ciencias } \\
\text { Ciencias de la salud }\end{array}$ & $\begin{array}{l}X \\
X\end{array}$ & $\begin{array}{l}X \\
X\end{array}$ & $\begin{array}{l}X \\
X\end{array}$ \\
\hline & $\begin{array}{l}\text { Ingeniería } \\
\text { Arquitectura }\end{array}$ & $\begin{array}{l}X \\
X\end{array}$ & $\begin{array}{l}X \\
X\end{array}$ & $\begin{array}{l}X \\
X\end{array}$ \\
\hline & $\begin{array}{l}\text { Ciencias sociales } \\
\text { Derecho }\end{array}$ & $\begin{array}{l}x \\
X\end{array}$ & $\begin{array}{l}x \\
x\end{array}$ & $\begin{array}{l}X \\
X\end{array}$ \\
\hline & Arte y humanidades & $x$ & $x$ & $x$ \\
\hline \multirow{3}{*}{$\begin{array}{l}\text { Perfil del } \\
\text { empleador }\end{array}$} & $\begin{array}{l}\text { Director recursos } \\
\text { humanos }\end{array}$ & $x$ & $x$ & $x$ \\
\hline & $\begin{array}{l}\text { Responsable } \\
\text { carreras } \\
\text { profesionales }\end{array}$ & $x$ & & $x$ \\
\hline & Directivo & $x$ & $x$ & $x$ \\
\hline
\end{tabular}

Algunos ejemplos de esta variedad de proyectos pueden verse en: a) Naval, C. y Arbués, E. (2018.) Hacer la Universidad en el espacio social. Concretamente en los capítulos: Costa, A. y Bañón, A., «Universidad y sociedad: la madurez de la acción y la forma del aprendizaje-servicio»; Ibarrola-García, S., «Innovación docente a través del aprendizaje-servicio: el caso de la asignatura "Aprendizaje de lenguas y atención a la diversidad lingüística"»; Carrica-Ochoa, S., «Evaluación de una experiencia de aprendizaje-servicio y del impacto positivo en la formación del alumnado universitario». b) Naval, C., Arbués, E. y Verea, N. (2016). El Aprendizaje-Servicio como recurso pedagógico en la Universidad. Revista inter-universitaria de investigación sobre discapacidad e interculturalidad. 


\subsection{Recogida de datos}

Se realizaron grupos focales para la recogida de datos, porque generan un discurso grupal para identificar distintas tendencias y regularidades en las opiniones (Barbour, 2013), y permite explorar las percepciones de los empleadores. Como muestra la tabla 1 , se realizaron tres grupos heterogéneos (de 4-5 personas cada grupo) considerando el sector en el que opera la empresa, el tipo de perfil del candidato que emplea y el perfil del empleador.

Los participantes recibieron por correo, con antelación, la hoja de información y consentimiento, y un breve documento con la definición del Aprendizaje-Servicio que de forma generalizada se utiliza en España -propuesta por Puig en 2009-, y que recogen en su web el Centre Promotor de Aprenentatge Servei, de Cataluña y la Fundación Zerbikas, de Euskadi, impulsores relevantes de esta metodología.

Se desarrolló una guía temática sustentada en la literatura y la experiencia en el tema de los investigadores, y que fue enviada para su revisión a siete expertos en Aprendizaje-Servicio. Cuatro de los expertos consultados están directamente implicados en la institucionalización de esta metodología en sus respectivas universidades y todos ellos participan activamente en grupos de investigación consolidados en los que el Aprendizaje-Servicio ocupa lugar relevante. Con base en las sugerencias menores realizadas por los expertos externos, se realizó la versión final de la guía, utilizada en los grupos focales, que abarca dos áreas: competencias profesionales más demandadas por los empleadores y Aprendizaje-Servicio e influencia en el perfil profesional.

Los grupos focales se realizaron en la universidad y se audio grabaron para su posterior transcripción textual para el análisis. Su duración fue de unos noventa minutos. El primer grupo focal, que sirvió de pilotaje, transcurrió de forma adecuada, razón por la que no hubo necesidad de hacer modificaciones en los siguientes. La entrevistadora fue una de las integrantes del proyecto del Ministerio al que se adscribía este estudio. Esta entrevistadora es profesora de la Facultad de Educación y Psicología de la Universidad de Navarra, y su investigación se desarrolla en la línea consolidada de Educación y Ciudadanía. La entrevistadora no conocía a los empleadores participantes en el estudio, dado que fueron propuestos y contactados por los directores de desarrollo de las distintas facultades de la Universidad de Navarra.

\subsection{Análisis de datos}

Se realizó un análisis de contenido inductivo. Dos personas independientemente, leyeron las transcripciones e identificaron unidades de significado que codificaron sin utilizar categorías predefinidas. En esta codificación inicial de las tres transcripciones (Krueger y Casey, 2015) se identificó que podían agruparse, clasificándolas en cuatro tipos buscando correspondencia con los cuatro temas del modelo de Cardona y García-Lombardía: interpersonales, negocio, eficacia personal externa, eficacia personal interna. Cada uno de estos cuatro temas tiene, a su vez, varias categorías: el tema de competencias interpersonales y el tema de competencias de negocio. Los otros dos temas incluyen, además de categorías, también subcategorías: el tema de competencias de eficacia personal externa y el tema de competencias de eficacia personal interna.

Se buscó la correspondencia de cada competencia profesional identificada en el análisis de contenido, con actitud de apertura para otras posibles competencias. Los empleadores 
de hecho, hacen referencia a muchos más matices, utilizando muchos conceptos (relativos a competencias profesionales) que no están en el modelo de Cardona y García-Lombardía, pero que se podrían incluir. Por ejemplo: a la subcategoría «autocontrol» de Cardona (tema «eficacia personal interna», categoría «autogobierno»), se han relacionado cuatro competencias profesionales identificadas en el análisis del discurso: autocontrol, que coincide con el modelo, y además: constancia, capacidad de sacrificio, esfuerzo y superación; categorías relativas a las competencias profesionales que destacaban los empleadores.

El análisis de los datos fue presentado a otros miembros del equipo de investigación para contrastar con ellos el proceso de codificación y agrupación, aportando mayor rigor. Se pidió a los otros miembros del equipo de investigación, que no habían participado en el proceso de codificación, que revisasen los códigos presentados y los datos que englobaban, como una forma de valorar la confiabilidad de los hallazgos. Posteriormente, se buscó en las transcripciones la frecuencia con que aparecían las categorías y subcategorías de cada tema de competencia profesional (de negocio, interpersonales y de eficacia personal -externa e interna-) y se registró.

Una vez hecho el recuento -para mostrar cuáles eran los temas de competencia profesional y las categorías más valoradas-, se calculó el porcentaje que cada tema y categoría representaba respecto del total. Del mismo modo, dentro de cada categoría, se calculó el porcentaje que representa cada una de las subcategorías que la integra.

En síntesis, los resultados se presentan en tablas que siguen la siguiente estructura:

Tabla 2. Presentación de resultados: estructura de las tablas e información contenida en ellas

\begin{tabular}{|l|l|c|c|c|c|}
\hline $\begin{array}{c}\text { Tema de } \\
\text { competencia } \\
\text { profesional }\end{array}$ & \multicolumn{2}{c}{ Demandadas por empleadores } & \multicolumn{2}{c|}{$\begin{array}{c}\text { Tema de } \\
\text { competencia } \\
\text { profesional }\end{array}$} \\
Porcentaje
\end{tabular}

También se presentan citas textuales de los empleadores que muestran los aspectos que destacan. 


\section{RESULTADOS Y DISCUSIÓN}

Los resultados muestran los temas de competencia profesional más valorados por los empleadores en los procesos de selección, así como las competencias profesionales más valoradas de cada categoría. Del mismo modo, se presentan los resultados obtenidos sobre las competencias que los empleadores consideran que se desarrollan mediante el Aprendizaje-Servicio, para mostrar una visión comparativa entre ambos aspectos.

Los empleadores, con sus propias palabras, demandan una gran variedad de competencias profesionales. Todas las competencias que mencionan pueden englobarse en los cuatro temas de competencias propuestos por Cardona y García-Lombardía: negocio, interpersonales, eficacia personal (externa e interna), aunque existen matices en las palabras/conceptos que utilizan los empleadores. A continuación se presentan los resultados sobre las competencias que más valoran los empleadores y su visión sobre si dichas competencias pueden desarrollarse mediante el Aprendizaje-Servicio por temas, categorías y subcategorías para mostrar los detalles, y facilitar la comparación entre lo que más valoran y su visión de Aprendizaje-Servicio.

La tabla 2 indica una perspectiva global de los temas de competencia profesional más demandados por los empleadores y su visión sobre si dichas competencias se pueden desarrollar mediante el Aprendizaje-Servicio.

La perspectiva global muestra que los temas de competencia profesional más valorados son el de eficacia personal interna; el interpersonal; seguido por el de eficacia personal externa; y, por último, negocio.

Tabla 3. Temas de competencia profesional más demandados por los empleadores del estudio y su perspectiva de la contribución del Aprendizaje-Servicio a su desarrollo

\begin{tabular}{|c|c|}
\hline Demandadas por los empleadores & Desarrolladas mediante ApS \\
\hline Eficacia personal interna $37.9 \%$ & Eficacia personal interna $43.6 \%$ \\
\hline Interpersonales $32.7 \%$ & Interpersonales $31.5 \%$ \\
\hline Eficacia personal externa $17.8 \%$ & Negocio $13 \%$ \\
\hline Negocio $11.5 \%$ & Eficacia personal externa $11.8 \%$ \\
\hline
\end{tabular}

Fuente: Elaboración propia.

Los empleadores consideran que los alumnos que han participado en Aprendizaje-Servicio, desarrollan primordialmente competencias de eficacia personal interna, seguidas por competencias interpersonales.

Esto concuerda con lo que ellos valoran como lo más importante en los candidatos que buscan. Pero si miramos más detalladamente los datos, observamos que existen matices. 
Tabla 4. Competencias eficacia personal interna, demandadas y desarrolladas en Aprendizaje-Servicio

\begin{tabular}{|c|c|c|c|c|c|c|}
\hline \multirow{2}{*}{$\begin{array}{l}\text { Competencias } \\
\text { eficacia personal } \\
\text { interna } \\
37.9 \%\end{array}$} & \multicolumn{3}{|c|}{ Demandadas por empleadores } & \multirow{2}{*}{$\begin{array}{c}\text { Competencias } \\
\text { eficacia } \\
\text { personal interna } \\
43.6 \%\end{array}$} & \multicolumn{2}{|c|}{$\begin{array}{l}\text { Desarrolladas mediante } \\
\qquad \mathrm{ApS}\end{array}$} \\
\hline & & Frecuencia & $\%$ & & Frecuencia & $\%$ \\
\hline Mejora personal & & & & Mejora personal & & \\
\hline $\begin{array}{c}\text { Autocrítica } \\
7.4 \%\end{array}$ & $\begin{array}{l}\text { Aceptarse } \\
\text { Asumir } \\
\text { Autocrítica } \\
\text { Autoexigencia }\end{array}$ & $\begin{array}{c}1 \\
7 \\
4 \\
4 \\
\text { Total } 16\end{array}$ & $\begin{array}{c}6.2 \\
43.8 \\
25 \\
25 \\
100\end{array}$ & $\begin{array}{c}\text { Autocrítica } \\
2.0 \%\end{array}$ & $\begin{array}{c}2 \\
0 \\
1 \\
0 \\
\text { Total } 3\end{array}$ & $\begin{array}{c}66.7 \\
0 \\
33.3 \\
0 \\
100\end{array}$ \\
\hline $\begin{array}{l}\text { Autoconoc. } \\
5.1 \%\end{array}$ & $\begin{array}{l}\text { Autoconoc. } \\
\text { Autoevaluación } \\
\text { Consciente }\end{array}$ & $\begin{array}{c}7 \\
2 \\
2 \\
\text { Total } 11\end{array}$ & $\begin{array}{l}63.6 \\
18.2 \\
18.2 \\
100\end{array}$ & $\begin{array}{l}\text { Autoconoc. } \\
\quad 4.6 \%\end{array}$ & $\begin{array}{c}6 \\
1 \\
0 \\
\text { Total } 7\end{array}$ & $\begin{array}{c}85.7 \\
14.3 \\
0 \\
100\end{array}$ \\
\hline $\begin{array}{l}\text { Aprendizaje } \\
20.7 \%\end{array}$ & $\begin{array}{l}\text { Apertura } \\
\text { Aprendizaje } \\
\text { Capac. } \\
\text { adaptación } \\
\text { cambio } \\
\text { Capac. } \\
\text { observación } \\
\text { Competente } \\
\text { Crecimiento } \\
\text { Enriquecer } \\
\text { Evaluación } \\
\text { Experiencia } \\
\text { Feedback } \\
\text { Flexibilidad } \\
\text { Habilidades } \\
\text { Hábito } \\
\text { Reflexionar } \\
\text { Sentido crítico }\end{array}$ & $\begin{array}{c}1 \\
0 \\
5 \\
3 \\
\\
\\
4 \\
3 \\
9 \\
2 \\
1 \\
2 \\
2 \\
4 \\
1 \\
4 \\
4 \\
\text { Total } 45 \\
\end{array}$ & $\begin{array}{c}2.2 \\
0 \\
11.1 \\
\\
6.7 \\
\\
8.9 \\
6.7 \\
20 \\
4.4 \\
2.2 \\
4.4 \\
4.4 \\
8.9 \\
2.2 \\
8.9 \\
8.9 \\
100\end{array}$ & $\begin{array}{l}\text { Aprendizaje } \\
28.5 \%\end{array}$ & $\begin{array}{c}9 \\
6 \\
3 \\
\\
3 \\
\\
2 \\
1 \\
3 \\
2 \\
6 \\
1 \\
2 \\
0 \\
3 \\
3 \\
0 \\
\text { Total } 43\end{array}$ & $\begin{array}{c}20.9 \\
14.0 \\
7 . \\
7 . \\
7 . \\
4.6 \\
2.2 \\
7.0 \\
4.6 \\
14.0 \\
2.2 \\
4.6 \\
0 \\
7.0 \\
7.0 \\
0 \\
100\end{array}$ \\
\hline Autogobierno & & & & Autogobierno & & \\
\hline $\begin{array}{c}\text { Toma decisiones } \\
1.4 \%\end{array}$ & $\begin{array}{l}\text { Analizar } \\
\text { Consejo } \\
\text { Tomar } \\
\text { decisiones }\end{array}$ & $\begin{array}{c}1 \\
1 \\
1 \\
\text { Total } 3\end{array}$ & $\begin{array}{l}33.3 \\
33.3 \\
33.3 \\
100\end{array}$ & $\begin{array}{c}\text { Toma decisiones } \\
0.7 \%\end{array}$ & $\begin{array}{c}0 \\
0 \\
1 \\
\text { Total } 1\end{array}$ & $\begin{array}{c}0 \\
0 \\
100 \\
100\end{array}$ \\
\hline $\begin{array}{l}\text { Autocontrol } \\
\quad 6 \%\end{array}$ & $\begin{array}{l}\text { Autocontrol } \\
\text { Capac. } \\
\text { sacrificio } \\
\text { Constante } \\
\text { Esfuerzo }\end{array}$ & $\begin{array}{c}4 \\
4 \\
4 \\
1 \\
\text { Total } 13\end{array}$ & \begin{tabular}{c|}
30.8 \\
30.8 \\
30.8 \\
7.6 \\
100
\end{tabular} & $\begin{array}{l}\text { Autocontrol } \\
6.7 \%\end{array}$ & $\begin{array}{c}5 \\
2 \\
1 \\
2 \\
\text { Total } 10\end{array}$ & $\begin{array}{c}50 \\
20 \\
10 \\
20 \\
100\end{array}$ \\
\hline $\begin{array}{l}\text { Equilibrio } \\
\text { emocional } \\
4.1 \%\end{array}$ & $\begin{array}{l}\text { I. emocional } \\
\text { Equilibrado/a } \\
\text { Estabilidad } \\
\text { carácter }\end{array}$ & $\begin{array}{c}8 \\
0 \\
1 \\
\text { Total } 9\end{array}$ & $\begin{array}{c}88.9 \\
0 \\
11.1 \\
100\end{array}$ & $\begin{array}{c}\text { Equilibrio } \\
\text { emocional } \\
2 \%\end{array}$ & $\begin{array}{c}2 \\
1 \\
0 \\
\text { Total } 3\end{array}$ & $\begin{array}{c}66.7 \\
33.3 \\
0 \\
100\end{array}$ \\
\hline
\end{tabular}




\begin{tabular}{|c|c|c|c|c|c|c|}
\hline $\begin{array}{c}\text { Integridad } \\
55.3 \%\end{array}$ & $\begin{array}{l}\text { Altruismo } \\
\text { Comprensión } \\
\text { Comprometido } \\
\text { Discreción } \\
\text { Disponibilidad } \\
\text { Ética } \\
\text { Generosidad } \\
\text { Honestidad } \\
\text { Humanidad } \\
\text { Humildad } \\
\text { Ideales } \\
\text { Identidad } \\
\text { Igualdad } \\
\text { Laboriosidad } \\
\text { Madurez } \\
\text { Prudencia } \\
\text { Respeto } \\
\text { Responsable } \\
\text { Sencillez } \\
\text { Sensibilizado } \\
\text { Sentido común } \\
\text { Capac. servicio } \\
\text { Sinceridad } \\
\text { Solidaridad } \\
\text { Tolerancia } \\
\text { Trascender } \\
\text { Valores } \\
\text { Virtudes }\end{array}$ & $\begin{array}{c}0 \\
0 \\
6 \\
1 \\
2 \\
2 \\
5 \\
2 \\
2 \\
3 \\
6 \\
8 \\
8 \\
2 \\
3 \\
14 \\
2 \\
1 \\
7 \\
5 \\
4 \\
1 \\
3 \\
6 \\
7 \\
8 \\
9 \\
0 \\
6 \\
7 \\
7 \\
\text { Total } \\
3\end{array}$ & $\begin{array}{c}0 \\
0 \\
5 \\
0.8 \\
1.7 \\
4.2 \\
1.7 \\
1.7 \\
2.5 \\
5.0 \\
6.7 \\
1.7 \\
2.5 \\
11.7 \\
1.7 \\
0.8 \\
5.8 \\
4.2 \\
3.3 \\
0.8 \\
2.5 \\
5.0 \\
5.8 \\
6.7 \\
7.5 \\
0 \\
5.0 \\
5.8 \\
100\end{array}$ & $\begin{array}{c}\text { Integridad } \\
55.6 \%\end{array}$ & $\begin{array}{c}1 \\
3 \\
12 \\
0 \\
0 \\
6 \\
1 \\
1 \\
8 \\
1 \\
3 \\
4 \\
2 \\
3 \\
4 \\
0 \\
2 \\
1 \\
1 \\
2 \\
1 \\
4 \\
2 \\
3 \\
3 \\
4 \\
7 \\
5 \\
\text { Total } 84\end{array}$ & $\begin{array}{c}1.2 \\
3.6 \\
14.3 \\
0 \\
0 \\
7.1 \\
1.2 \\
1.2 \\
9.5 \\
1.2 \\
3.6 \\
4.8 \\
2.4 \\
3.6 \\
4.8 \\
0 \\
2.4 \\
1.2 \\
1.2 \\
2.4 \\
1.2 \\
4.8 \\
2.4 \\
3.6 \\
3.6 \\
4.8 \\
8.4 \\
6 \\
100\end{array}$ \\
\hline
\end{tabular}

La competencia más valorada por los empleadores y que más piensan que se desarrolla desde Aprendizaje-Servicio es la integridad. En el marco teórico de Cardona y GarcíaLombardía se define la integridad como la capacidad de comprometerse de manera recta y honrada ante cualquier situación.

Los empleadores coinciden en señalar que la capacidad de compromiso supone un valor para la organización, ya que genera la vinculación que las organizaciones buscan a largo plazo con sus empleados. Cuantas más personas comprometidas integren la organización, más comprometida llegará a ser la organización, como muestra la siguiente cita:

Las organizaciones están compuestas de personas con un nivel personal de compromiso. Personas comprometidas construyen organizaciones comprometidas (G2E4).

Por tanto, el logro de este compromiso hacia la organización depende de que las personas que la integran posean integridad o capacidad de compromiso con un objetivo común valioso; esto se traduce en poseer sentido de misión, combinado con la capacidad de conjugar los propios intereses con los de la organización, siendo capaces de trascenderlos pero sin perder la autenticidad personal (Yuan et al., 2018).

No obstante, los empleadores consultados precisan que la organización tiene un papel fundamental al generar compromiso en los empleados. Sin esta iniciativa de la organización, ese compromiso positivo llegaría a perderse por la falta de coherencia organizacional: 
Generar compromiso es responsabilidad de la organización Si el compromiso se queda en el papel, en las misiones, en la organización formal, pero no impregna las relaciones cotidianas, genera incoherencia interna y, en consecuencia, desmotivación y desilusión (G2E4).

En definitiva, contar con personas comprometidas cuyo compromiso se ve reforzado por políticas organizativas comprometidas, genera excelencia organizacional estratégica, intratégica y ética (Cheong et al., 2016; Latta, 2020; Yuan et al., 2018).

Por otra parte, para generar el anhelado compromiso personal y organizacional, es necesaria además una voluntad estable hacia el mismo, apoyada en una toma de decisiones reflexiva, coherente e íntegra. Esta toma de decisiones -asentada en el pensamiento crítico-, aporta la capacidad para reflexionar sobre el proyecto organizacional y, si este es valioso, concluir en una comprensión comprometida (Van Der Westhuizen et al., 2012).

Sin embargo, nuestros empleadores, no valoran la competencia de toma de decisiones como parte del perfil clave al seleccionar ni piensan que se desarrolle en Aprendizaje-Servicio. Esta escasa valoración puede deberse a que en el estudio indagamos su percepción sobre las competencias profesionales para la empleabilidad de los universitarios. Estos candidatos acaban de finalizar sus estudios y habitualmente acceden a puestos en los que esta competencia no es tan requerida como otras, por ejemplo las competencias comunicativas, el pensamiento crítico, la creatividad, la resiliencia o la iniciativa (Consejo de la Unión Europea, 2018; OECD, 2015 y 2017).

El segundo tema de competencia profesional más valorado por los empleadores y que más piensan que se desarrolla desde Aprendizaje-Servicio es la competencia interpersonal. No obstante, no hay coincidencia en el orden de preferencia entre las categorías de competencias que lo conforman.

Tabla 5. Competencias interpersonales demandadas y desarrolladas en Aprendizaje-Servicio

\begin{tabular}{|c|l|c|c|c|c|c|}
$\begin{array}{c}\text { Competencias } \\
\text { interpersonales } \\
32.7 \%\end{array}$ & \multicolumn{2}{|c|}{ Demandadas por los empleadores } & $\begin{array}{l}\text { Competencias } \\
\text { interpersonales } \\
31.5 \%\end{array}$ & \multicolumn{2}{c|}{$\begin{array}{l}\text { Desarrolladas } \\
\text { mediante ApS }\end{array}$} \\
& & Frec. & $\%$ & & Frec. & $\%$ \\
\hline & & 9 & 14.1 & Comunicación & 1 & 3.8 \\
\hline Comunicación & Asertividad & 30 & 46.9 & $23.9 \%$ & 16 & 61.6 \\
& Comunicación & 18 & 28.1 & & 5 & 19.2 \\
& Escuchar & 1 & 1.6 & & 0 & 0 \\
& Expresar ideas & 2 & 3.1 & & 4 & 15.4 \\
& Hablar público & 2 & 3.1 & & 0 & 0 \\
& Transmitir & 2 & 3.1 & & 0 & 0 \\
& Transparencia & Total 64 & 100 & & Total 26 & 100 \\
\hline Gestión & Afrontar & 7 & 35 & Gestión & 4 & 23.5 \\
conflictos & Detectar & 3 & 15 & conflictos & 1 & 5.9 \\
$10.7 \%$ & Relativizar & 4 & 20 & $15.6 \%$ & 4 & 23.5 \\
& Resolver & 6 & 30 & & 8 & 47.1 \\
& & Total 20 & 100 & & Total 17 & 100 \\
\hline
\end{tabular}




\begin{tabular}{|c|c|c|c|c|c|c|}
\hline $\begin{array}{l}\text { Carisma } \\
24.6 \%\end{array}$ & $\begin{array}{l}\text { Confianza } \\
\text { Seguimiento } \\
\text { Coordinar } \\
\text { Ejemplaridad } \\
\text { Exigir } \\
\text { Liderazgo } \\
\text { Motivación }\end{array}$ & $\begin{array}{c}7 \\
12 \\
2 \\
2 \\
13 \\
3 \\
7 \\
\text { Total } 46\end{array}$ & $\begin{array}{c}15.2 \\
26.1 \\
4.3 \\
4.3 \\
28.3 \\
6.5 \\
15.2 \\
100\end{array}$ & $\begin{array}{l}\text { Carisma } \\
29.4 \%\end{array}$ & $\begin{array}{c}2 \\
18 \\
1 \\
1 \\
0 \\
7 \\
3 \\
\text { Total } 32\end{array}$ & $\begin{array}{c}6.2 \\
56.3 \\
3.1 \\
3.1 \\
0 \\
21.9 \\
9.4 \\
100\end{array}$ \\
\hline $\begin{array}{c}\text { Empowerment } \\
7 \%\end{array}$ & $\begin{array}{l}\text { Delegar } \\
\text { Implicar }\end{array}$ & $\begin{array}{c}11 \\
2 \\
\text { Total } 13\end{array}$ & $\begin{array}{l}84.6 \\
15.4 \\
100\end{array}$ & $\begin{array}{c}\text { Empowerment } \\
2.8 \%\end{array}$ & $\begin{array}{c}0 \\
3 \\
\text { Total } 3\end{array}$ & $\begin{array}{c}0 \\
100 \\
100\end{array}$ \\
\hline $\begin{array}{r}\text { Coaching } \\
4,8 \%\end{array}$ & $\begin{array}{l}\text { Acompañamiento } \\
\text { Orientar } \\
\text { Avance }\end{array}$ & $\begin{array}{c}3 \\
5 \\
1 \\
\text { Total } 9\end{array}$ & $\begin{array}{l}33.3 \\
55.6 \\
11.1 \\
100\end{array}$ & $\begin{array}{r}\text { Coaching } \\
0 \%\end{array}$ & $\begin{array}{l}0 \\
0 \\
0\end{array}$ & $\begin{array}{l}0 \\
0 \\
0\end{array}$ \\
\hline $\begin{array}{c}\text { Trabajo equipo } \\
18,7 \%\end{array}$ & $\begin{array}{l}\text { Acogida } \\
\text { Colaborar } \\
\text { Convivir } \\
\text { Empatía } \\
\text { Mejora clima/ } \\
\text { entorno } \\
\text { Trabajo equipo } \\
\text { Sinergias }\end{array}$ & $\begin{array}{c}1 \\
1 \\
5 \\
18 \\
0 \\
7 \\
3 \\
\text { Total } 35\end{array}$ & $\begin{array}{c}2.9 \\
2.9 \\
14.2 \\
51.4 \\
0 \\
20 \\
8.6 \\
100\end{array}$ & $\begin{array}{c}\text { Trabajo equipo } \\
28.4 \%\end{array}$ & $\begin{array}{c}0 \\
1 \\
1 \\
17 \\
2 \\
8 \\
2 \\
\text { Total } 31\end{array}$ & $\begin{array}{c}0 \\
3.2 \\
3.2 \\
54.8 \\
6.5 \\
25.8 \\
6.5 \\
100\end{array}$ \\
\hline
\end{tabular}

Los empleadores al realizar una selección, prefieren que los candidatos posean la competencia de comunicación, seguida del carisma y después el trabajo en equipo. Piensan que quienes participan en Aprendizaje-Servicio desarrollan primero el carisma, después el trabajo en equipo y en tercer lugar la comunicación.

Como se ha visto, la competencia que los empleadores de nuestro estudio más valoran en los candidatos, es la integridad o capacidad para comprometerse. Afirmaban que es clave que el compromiso personal se refuerce e incluso se genere desde la organización. Lograr organizaciones capaces de generar compromiso depende, en gran medida, del perfil de liderazgo o carisma de las personas con responsabilidades directivas (Cheong et al., 2016). El carisma es la capacidad de lograr el compromiso de los colaboradores, inspirando su confianza, dando sentido a su trabajo y motivándoles a conseguir sus objetivos (Cardona y García-Lombardía, 2005). Esta competencia está entre las consideradas clave por los empleadores; y es la que consideran que más se consigue a través de Aprendizaje-Servicio de entre todas las interpersonales. El carisma supone (Cheong et al., 2016):

- Una alta empatía, para colocarse en el lugar de sus colaboradores y comprender su punto de vista.

- Una comunicación eficaz y transparente para transmitir que lo divergente -si es constructivo, y no contraviene los valores y principios expresados en las misiones-, enriquece la organización.

- La promoción de la participación activa y creativa para encauzar efectivamente el respeto expresado hacia las opiniones y aportaciones de los miembros de la organización. 
- La apertura, honestidad, rectitud e integridad del directivo para entender y respetar la riqueza que encierra la diversidad de enfoques.

En consecuencia, en los procesos de selección resultaría adecuado valorar la competencia carisma, ya que personas con este perfil podrían llegar a desempeñar labores directivas a medio-largo plazo en las organizaciones en las que fueran contratados. Es decir, la competencia carisma aporta a los candidatos un valor que puede proyectarse progresivamente en la carrera profesional dando acceso -conjugándola con otras variables- a puestos de dirección (Cheong et al., 2016). Los empleadores de nuestro estudio advierten esta relación, pues el carisma es otra de las competencias valoradas al seleccionar. Por otra parte, opinan que esta competencia se refuerza participando en Aprendizaje-Servicio.

El tema de competencia profesional valorado por los empleadores, en tercer lugar, es la eficacia personal externa. Con Aprendizaje-Servicio, según nuestros empleadores, esta se desarrolla en cuarto lugar, por detrás del tema de competencia profesional de negocio.

Tabla 6. Competencias eficacia personal externa demandadas y desarrolladas en Aprendizaje-Servicio

\begin{tabular}{|c|c|c|c|c|c|c|}
\hline \multirow{2}{*}{$\begin{array}{l}\text { Competencias } \\
\text { eficacia } \\
\text { personal } \\
\text { externa } \\
17.8 \%\end{array}$} & \multicolumn{3}{|c|}{ Demandadas por empleadores } & \multirow{2}{*}{$\begin{array}{l}\text { Competencias } \\
\text { eficacia personal } \\
\text { externa } \\
11.8 \%\end{array}$} & \multicolumn{2}{|c|}{$\begin{array}{l}\text { Desarrolladas } \\
\text { mediante ApS }\end{array}$} \\
\hline & & Frec. & $\%$ & & Frec. & $\%$ \\
\hline Proactividad & & & & Proactividad & & \\
\hline $\begin{array}{c}\text { Iniciativa } \\
47.1 \%\end{array}$ & $\begin{array}{l}\text { Acometer } \\
\text { Apasionamiento } \\
\text { Automotivación } \\
\text { Autonomía } \\
\text { Creatividad } \\
\text { Curiosidad } \\
\text { Iniciativa } \\
\text { Dinámico } \\
\text { Empuje } \\
\text { Energía } \\
\text { Implicación } \\
\text { Innovación } \\
\text { Participación } \\
\text { Proactivo } \\
\text { Promover } \\
\text { Reinventarse } \\
\text { Responsabilidad } \\
\text { Transformar }\end{array}$ & $\begin{array}{c}5 \\
5 \\
1 \\
1 \\
3 \\
1 \\
3 \\
1 \\
1 \\
2 \\
3 \\
3 \\
9 \\
5 \\
1 \\
1 \\
2 \\
1 \\
\text { Total } 48\end{array}$ & $\begin{array}{l}10.4 \\
10.4 \\
2.1 \\
2.1 \\
6.2 \\
2.1 \\
6.2 \\
2.1 \\
2.1 \\
4.2 \\
6.2 \\
6.2 \\
18.7 \\
10.4 \\
2.1 \\
2.1 \\
4.2 \\
2.1 \\
100\end{array}$ & $\begin{array}{l}\text { Iniciativa } \\
70.7 \%\end{array}$ & $\begin{array}{c}4 \\
2 \\
0 \\
0 \\
2 \\
0 \\
2 \\
0 \\
0 \\
1 \\
4 \\
0 \\
6 \\
2 \\
0 \\
1 \\
5 \\
0 \\
\text { Total } 29\end{array}$ & $\begin{array}{c}13.8 \\
6.9 \\
0 \\
0 \\
6.9 \\
0 \\
6.9 \\
0 \\
0 \\
3.4 \\
13.8 \\
0 \\
20.7 \\
6.9 \\
0 \\
3.4 \\
17.2 \\
0 \\
100\end{array}$ \\
\hline
\end{tabular}




\begin{tabular}{|c|c|c|c|c|c|c|}
\hline $\begin{array}{l}\text { Optimismo } \\
25.5 \%\end{array}$ & $\begin{array}{l}\text { No agobiarse } \\
\text { Optimismo } \\
\text { Positivo } \\
\text { Realismo } \\
\text { Resiliencia } \\
\text { Resistencia } \\
\text { frustración } \\
\text { Resistencia } \\
\text { fracaso }\end{array}$ & $\begin{array}{c}1 \\
0 \\
1 \\
1 \\
5 \\
12 \\
6\end{array}$ & $\begin{array}{c}3.8 \\
0 \\
3.8 \\
3.8 \\
19.2 \\
\\
46.2 \\
\\
\\
23.1 \\
\\
\\
\end{array}$ & $\begin{array}{l}\text { Optimismo } \\
19.5 \%\end{array}$ & $\begin{array}{c}0 \\
2 \\
0 \\
2 \\
0 \\
3 \\
1 \\
\\
\text { Total } 8\end{array}$ & $\begin{array}{c}0 \\
25 \\
0 \\
25 \\
0 \\
37.5 \\
\\
12.5 \\
\\
100\end{array}$ \\
\hline $\begin{array}{c}\text { Ambición } \\
2 \%\end{array}$ & $\begin{array}{l}\text { Competitivo } \\
\text { Determinación }\end{array}$ & $\begin{array}{c}1 \\
1 \\
\text { Total } 2\end{array}$ & $\begin{array}{c}50 \\
50 \\
100\end{array}$ & $\begin{array}{l}\text { Ambición } \\
0 \%\end{array}$ & $\begin{array}{l}0 \\
0\end{array}$ & $\begin{array}{l}0 \\
0\end{array}$ \\
\hline $\begin{array}{l}\text { Gestión } \\
\text { personal }\end{array}$ & & & & Gestión personal & & \\
\hline $\begin{array}{l}\text { Gestión } \\
\text { tiempo } \\
11.8 \%\end{array}$ & $\begin{array}{l}\text { Puntual } \\
\text { Ordenado } \\
\text { Organizarse } \\
\text { Priorizar }\end{array}$ & $\begin{array}{c}3 \\
2 \\
4 \\
3 \\
\text { Total } 12\end{array}$ & $\begin{array}{c}25.0 \\
16.7 \\
33.3 \\
25 \\
100\end{array}$ & $\begin{array}{l}\text { Gestión tiempo } \\
4.9 \%\end{array}$ & $\begin{array}{c}2 \\
0 \\
0 \\
0 \\
\text { Total } 2\end{array}$ & $\begin{array}{c}100 \\
0 \\
0 \\
0 \\
100\end{array}$ \\
\hline $\begin{array}{c}\text { Gestión } \\
\text { información } \\
7.8 \%\end{array}$ & $\begin{array}{l}\text { Gest. info. } \\
\text { Identificar } \\
\text { Sintetizar }\end{array}$ & $\begin{array}{c}2 \\
5 \\
1 \\
\text { Total } 8\end{array}$ & $\begin{array}{c}25 \\
62.5 \\
12.5 \\
100\end{array}$ & $\begin{array}{l}\text { Gestión } \\
\text { información } \\
4.9 \%\end{array}$ & $\begin{array}{c}1 \\
1 \\
0 \\
\text { Total } 2\end{array}$ & $\begin{array}{c}50 \\
50 \\
0 \\
100\end{array}$ \\
\hline $\begin{array}{c}\text { Gestión estrés } \\
5.9 \%\end{array}$ & $\begin{array}{l}\text { Equilibrado/a } \\
\text { Gestionar estrés/ } \\
\text { tensión }\end{array}$ & $\begin{array}{c}4 \\
2 \\
\text { Total } 6\end{array}$ & $\begin{array}{l}66.7 \\
33.3 \\
100\end{array}$ & $\begin{array}{l}\text { Gestión estrés } \\
0 \%\end{array}$ & $\begin{array}{l}0 \\
0\end{array}$ & $\begin{array}{l}0 \\
0\end{array}$ \\
\hline
\end{tabular}

En los procesos de selección, los empleadores valoran la iniciativa y el optimismo, por encima de otras competencias de esta categoría. Con Aprendizaje-Servicio manifiestan lo mismo. Concretamente para estos empleadores, quienes participan en esta metodología de aprendizaje, desarrollan exponencialmente la competencia iniciativa. La competencia optimismo se posee de manera similar a quienes no han hecho estas actividades. Los empleadores refieren:

[El Aprendizaje-Servicio aporta] Iniciativa, ese empuje que le lleva a buscar soluciones, que es la parte creativa (G1E2).

Para Cardona y García-Lombardía (2005), la iniciativa es la capacidad de mostrar un comportamiento emprendedor, iniciando e impulsando los cambios necesarios con energía y responsabilidad personal.

Mirando de nuevo a la integridad o capacidad de compromiso como competencia referida por nuestros empleadores como principal al contratar, se puede afirmar que la iniciativa adquiere relevancia para consolidar y ampliar el valor que supone el compromiso. El compromiso supone, no solo la identificación y lealtad con la misión, la visión y los valores de la organización. También se requiere la iniciativa para participar responsable, activa y creativamente 
en la mejora de la organización. Por otra parte, quienes tienen la responsabilidad de generar compromiso, han de estar abiertos a la iniciativa personal para aceptar propuestas, impulsar proyectos y acometer cambios, siendo esta iniciativa la base para la innovación. Se trataría de guiar y conjugar positiva y constructivamente -con flexibilidad-la necesaria visión personal con la visión organizacional (Van Der Westhuizen et al., 2012).

No obstante, si quienes poseen un alto compromiso se encuentran con una organización poco comprometida o incoherente, deberán apoyarse en otra de las competencias profesionales que los empleadores valoran al contratar y que desarrollan quienes participan en Aprendizaje-Servicio: el optimismo o la capacidad de ver el lado positivo de la realidad, tener fe en las propias responsabilidades y afrontar las dificultades con entusiasmo (Cardona y García-Lombardía, 2005). Esta competencia ayudará a los futuros profesionales a resistir en caso de presentarse un entorno organizacional menos comprometido:

\footnotetext{
Que sea una persona con resiliencia, o sea, con tolerancia a la frustración, resistente al fracaso (G1E1).

[Poseen] resiliencia, actitud positiva ante los problemas. Es como determinación. Es la capacidad de superación, sí, de seguir adelante, ¿no? (G3E4).
}

Finalmente, en los procesos de selección, los empleadores de nuestro estudio valoran el tema de competencia profesional de negocio. Como se señaló, opinan que quienes participan en Aprendizaje-Servicio desarrollan las competencias de negocio por delante de las competencias de eficacia personal externa, que valoran al contratar en tercer lugar. Tabla 7. Competencias de negocio demandadas
y desarrolladas en Aprendizaje-Servicio

\begin{tabular}{|c|c|c|c|c|c|c|}
\hline \multirow{2}{*}{$\begin{array}{l}\text { Competencias } \\
\text { negocio } \\
11.5 \%\end{array}$} & \multicolumn{3}{|c|}{ Demandadas por los empleadores } & \multirow{2}{*}{$\begin{array}{l}\text { Competencias } \\
\text { negocio } \\
13 \%\end{array}$} & \multicolumn{2}{|c|}{$\begin{array}{l}\text { Desarrolladas } \\
\text { mediante ApS }\end{array}$} \\
\hline & & Frecuencia & $\%$ & & Frecuencia & $\%$ \\
\hline $\begin{array}{l}\text { Visión negocio } \\
18.2 \%\end{array}$ & $\begin{array}{l}\text { Competitivo } \\
\text { Implantar } \\
\text { Objetivo común } \\
\text { Rentabilidad } \\
\text { Resultado }\end{array}$ & $\begin{array}{c}3 \\
2 \\
2 \\
2 \\
3 \\
\text { Total } 12\end{array}$ & $\begin{array}{c}25 \\
16.7 \\
16.7 \\
16.7 \\
25 \\
100\end{array}$ & $\begin{array}{l}\text { Visión negocio } \\
15.6 \%\end{array}$ & $\begin{array}{c}0 \\
0 \\
5 \\
1 \\
2 \\
\text { Total } 7\end{array}$ & $\begin{array}{c}0 \\
71.4 \\
12.5 \\
25 \\
100\end{array}$ \\
\hline $\begin{array}{l}\text { Visión } \\
\text { organización } \\
30.3 \%\end{array}$ & $\begin{array}{l}\text { Estrategia } \\
\text { Interdisciplinariedad } \\
\text { Polivalencia } \\
\text { Sentido misión } \\
\text { Visión global }\end{array}$ & $\begin{array}{c}4 \\
1 \\
2 \\
0 \\
13 \\
\text { Total } 20\end{array}$ & $\begin{array}{c}20 \\
5 \\
10 \\
0 \\
65 \\
100\end{array}$ & $\begin{array}{l}\text { Visión } \\
\text { organización } \\
48.9 \%\end{array}$ & $\begin{array}{c}2 \\
6 \\
2 \\
6 \\
6 \\
\text { Total } 22\end{array}$ & $\begin{array}{c}9.1 \\
27.3 \\
9.1 \\
27.3 \\
27.3 \\
100\end{array}$ \\
\hline $\begin{array}{l}\text { Orientación al } \\
\text { cliente } \\
21.2 \%\end{array}$ & $\begin{array}{l}\text { Conseguir } \\
\text { Eficacia } \\
\text { Interrelación }\end{array}$ & $\begin{array}{c}7 \\
1 \\
6 \\
\text { Total } 14\end{array}$ & $\begin{array}{c}50 \\
7.1 \\
42.9 \\
100\end{array}$ & $\begin{array}{l}\text { Orientación al } \\
\text { cliente } \\
13.3 \%\end{array}$ & $\begin{array}{c}0 \\
0 \\
6 \\
\text { Total } 6\end{array}$ & $\begin{array}{c}0 \\
0 \\
100 \\
100\end{array}$ \\
\hline
\end{tabular}




\begin{tabular}{|l|l|c|c|l|c|c|}
\hline Negociación & Acuerdos & 1 & 14.3 & Negociación & 1 & 25 \\
$10.6 \%$ & Negociación & 5 & 71.4 & $8.9 \%$ & 3 & 75 \\
& Ceder & $\begin{array}{c}1 \\
\text { Total } 7\end{array}$ & 100 & & 0 & 0 \\
& & 3 & 25 & Networking & 3 & 50 \\
Networking & Alianzas & 0 & 0 & $13.3 \%$ & 0 & 0 \\
$18.2 \%$ & Networking & 2 & 16.7 & & 0 & 0 \\
& Redes & 7 & 58.3 & & 3 & 50 \\
& Relaciones & Total 12 & 100 & & Total 6 & 100 \\
& & 1 & 100 & Gestión & 0 & 0 \\
\hline Gestión & Gestión recursos & & & recursos $0 \%$ & & \\
recursos & & & & & & \\
$1.5 \%$ & & & & & & \\
\hline
\end{tabular}

Fuente: Elaboración propia.

Dentro de esta categoría, los empleadores consideran que los candidatos han de poseer en primer lugar, visión de la organización, seguida de orientación al cliente y tras ella, visión de negocio. Opinan que al colaborar en Aprendizaje-Servicio se desarrolla también primordialmente la visión de la organización, después la visión de negocio y luego la orientación al cliente. Es decir, coinciden en considerar central entre las competencias de negocio, la visión de la organización.

Valorar en primer lugar la visión de la organización dentro de las competencias de negocio, coincide con su apuesta por un candidato con integridad o capacidad de compromiso.

En nuestro modelo competencial de referencia, se define la visión de la organización como la capacidad de valorar la empresa más allá de los límites de la propia función, comprender la interrelación entre las distintas unidades y desarrollar la cooperación interfuncional. Esta competencia supone elevar la mirada de la propia visión personal hacia el objetivo común de la organización. Sin olvidar que, tanto la visión personal como la visión organizacional, han de mirar a la misión y los valores de la organización, dotando de coherencia y unidad a la actuación personal y organizacional (Yuan et al., 2018).

En este sentido, podría indicarse que la competencia visión de la organización, contribuye a fortalecer la capacidad de compromiso que nuestros empleadores valoran en los procesos de selección y que desarrollan los alumnos que participan en Aprendizaje-Servicio. Así se manifiesta en el énfasis que en estas ideas realizan los empleadores:

Una persona que se ha comprometido tiene una sensibilidad especial. Las acciones sociales te dignifican un montón, salen mucho de sí mismos [...]. Es una manera de acabar con el individualismo tan brutal que existe hoy día (G1E1).

[Participar en Aprendizaje-Servicio] te prepara para adquirir un sentido de misión (G1E3).

En definitiva: los resultados muestran que el perfil competencial descrito como idóneo por los empleadores, se fomenta participando en Aprendizaje-Servicio en la mayor parte de las competencias, pero presenta algunas diferencias en el orden de preferencia. 


\section{LIMITACIONES DEL ESTUDIO}

A la hora de considerar los resultados debe tenerse en cuenta que los participantes eran empleadores de egresados de una universidad. Sería interesante indagar la visión de los empleadores de otras universidades que también llevan a cabo el Aprendizaje-Servicio.

\section{IMPLICACIONES DEL ESTUDIO}

El estudio realizado sugiere que el Aprendizaje-Servicio contribuye a desarrollar competencias que los empleadores consideran importantes y, por ello, estos lo valoran en los procesos de selección. Por tanto, resultaría adecuado:

1. Extender esta metodología en las universidades como vía para reforzar la cualificación competencial de los universitarios y, en consecuencia, su empleabilidad. Esto también ayudaría a acercar el mundo laboral a la universidad.

2. Ampliar la formación de los empleadores sobre esta metodología y potenciar que en las organizaciones se acepten alumnos que estén participando en programas de AprendizajeServicio durante sus estudios universitarios. Así mismo fomentar que los empleadores valoren más el Aprendizaje-Servicio y lo consideren al plantearse su incorporación a la organización.

3. Finalmente resultaría indicado abrir más vías de colaboración entre universidad y entidades empresariales, para seguir indagando en la transferencia de las competencias desarrolladas mediante el Aprendizaje-Servicio al mundo laboral.

\section{CONCLUSIONES}

1. Respecto del objetivo general del estudio, los resultados indican que el perfil profesional competencial para la empleabilidad de los universitarios -descrito como idóneo por los empleadores participantes en nuestro estudio- se fomenta con el Aprendizaje-Servicio, en la mayor parte de las competencias. Se puede inferir, por tanto, que estos universitarios podrían llegar a ser candidatos más atractivos en los procesos de selección, mejorando en consecuencia su empleabilidad; siempre y cuando los empleadores conozcan la contribución a la mejora del perfil competencial que puede conllevar el uso de esta metodología.

2. En torno del primer objetivo específico, los empleadores de nuestro estudio consideran que el perfil idóneo para la empleabilidad es el que posee las siguientes competencias: integridad (eficacia personal interna); habilidades de comunicación, carisma y capacidad de trabajo en equipo (interpersonales); iniciativa y optimismo (eficacia personal externa), y visión de la organización (negocio). Este perfil se ajusta al marco teórico de Cardona. Parece que existe una base que sugiere que todas las competencias que valoran los empleadores, en realidad, tienen su lugar en el modelo de Cardona. Indagar esta cuestión no estaba en los objetivos del estudio, pero los resultados muestran la solidez del marco conceptual. 
3. Referente al segundo objetivo específico, los resultados muestran que el AprendizajeServicio refuerza el perfil competencial que los empleadores de nuestro estudio demandan a los universitarios en los procesos de selección.

4. En nuestro estudio también comprobamos que a lo largo del proceso, los empleadores incrementaron su conocimiento del Aprendizaje-Servicio. De este modo, percibimos que a medida que avanzaban en este conocimiento, descubrían su potencial y destacaban su valor para incrementar las oportunidades de aprendizaje, diálogo y compromiso y que, por tanto, esta metodología puede aportar nuevos caminos para la adquisición de competencias profesionales. En este sentido, sería adecuado propiciar y aumentar este tipo de canales de aprendizaje en que el alumno universitario desarrolla competencias transversales al margen de las estrictamente curriculares.

\section{REFERENCIAS}

Barbour, R. (2013). Los grupos de discusión en investigación cualitativa. España: Ediciones Morata.

Blokker, R.; Akkermans, J.; Tims, M.; Jansen, P. \& Khapova, S. (2019). Building a sustainable start: The role of career competencies, career success, and career shocks in young professionals employability. Journal of Vocational Behavior, 112, 172-184. https://doi.org/10.1016/j. jvb.2019.02.013

Boyatzis, R. (1982). The competent manager. A model for effective performance. John Willey \& Sons.

Boyatzis, R. (2011). Managerial and Leadership Competencies. A behavioural approach to emotional, social and cognitive intelligence. Vision, 15(2), 91-100. https://doi. org/10.1177/097226291101500202

Cardona, P. y García-Lombardía, P. (2005). Cómo desarrollar las competencias de liderazgo. España: EUNSA.

Cedefop, European Training Foundation (ETF), UNESCO \& the UNESCO Institute for Lifelong Learning (2017). Global Inventory of Regional and National Qualifications Frameworks 2017. Volume I: Thematic Chapters. https:// www.cedefop.europa.eu/en/publications-andresources/publications/2221

Comisión Europea (2010). EUROPA 2020 Una estrategia para un crecimiento inteligente, sostenible e integrador. http:/ / eur-lex.europa.eu/legal-content/ES/TXT/?uri=celex:52010DC2020

Cheong, M., Spain, S. M., Yammarino, F. J. \& Yun, S. (2016). Two faces of empowering leadership: Enabling and burdening. The Leadership Quarterly, 27(4), 602-616 https:/ / doi. org/10.1016/j.leaqua.2016.01.006

Gay, P., Salaman, G. \& Reys, B. (1996). The conduct of management and the management of the conduct. Journal of Management Studies, 33(3), 263-282. https://doi. org/10.1111/j.1467-6486.1996.tb00802.x

Giorgi, G. (2013). Organizational Emotional Intelligence: development of a model. International Journal ofOrganizational Analysis, 21(1), 4-18 https:/ / doi.org/10.1108/19348831311322506 
Giorgi, G. \& Majer, V. (2012). Intelligenza organizzativa: competenze emotive ed organizzative per l'eccelenza. Giunti OS, Organizzazioni Speciali. Development of a Model. International Journal of Organizational Analysis, 21(1), 4-18.

Goleman, D.; Boyatzis, R.; McKee, A. \& Rock, E. (2002). Primal leadership. Harvard Business Review, 78(2), 78-90 https:/ / doi.org/10.1002/hrdq.1063

Hora, M. T.; Benbow, R. J. \& Oleson, A. K. (2016). Beyond the Skills Gap. Preparing College Students for Life and Work. Harvard Education Press.

Humburg, M.; Van der Velden, R. \& Verhagen, A. (2013). The employability of higher education graduates: the employers' perspective. https://publications.europa.eu/en/publicationdetail/-/ publication/ecbcc42d-349e-4903-a844-9820680baa1a/language-en

Jagla, V. M. \& Tice, K. C. (2019). Educating Teachers \& Tomorrow's Students through ServiceLearning Pedagogy. Information Age Publishing, INC.

Krueger, R. A. \& Casey, M. A. (2015). Focus Groups. A Practical Guide for Applied Research. SAGE.

Latta, G. F. (2020). A complexity analysis of organizational culture, leadership and engagement: integration, differentiation and fragmentation. International Journal of Leadership in Education, 23(3), 274-299. https:// doi.org/10.1080/13603124.2018.1562095

Miller, A. L.; Rocconi, M. \& Dumford, A. D. (2018). Focus on the finish line: does high-impact practice participation influence career plans and early job attainment? The International Journal of Higher Education Research, 75(3), 489-506. https://doi.org/10.1007/s10734$\underline{017-0151-\mathrm{Z}}$

Morone, M.; Giorgi, G. \& Fiz Pérez, J. (2016). Emotional and organizational competency for success at work: a review. Calitatea, 17(152), 120-125.

Naval, C.; Arbués, E. y Verea, N. (2016). El Aprendizaje-Servicio como recurso pedagógico en la Universidad. En Educación y Diversidad. Revista inter-universitaria de investigación sobre discapacidad e interculturalidad, 10(2) julio-diciembre 2016. Zaragoza: Prensas de la Universidad de Zaragoza.

OECD (2015). OECD Skills Outlook 2015: Youth, Skills and Employability, OECD. https://doi. org/10.1787/9789264234178-en

OECD (2017). OECD Skills Outlook 2017. Skills and Global Value Chains. Paris: OECD. https:// doi.org/10.1787/9789264273351-en

Pineda-Herrero, P.; Ciraso-Cali, A. y Armijos-Yambay, M. (2018). Competencias para la empleabilidad de los titulados en Pedagogía, Psicología y Psicopedagogía: un estudio comparativo entre empleadores y titulados. Revista Española de Pedagogía, 76(270), 313333. https: / / doi.org/10.22550/ REP76-2-2018-06

Puig, J.M. (coord.) (2009). Aprendizaje-servicio (ApS). Educación y compromiso cívico. España: Graó.

Rios, J. A.; Ling, G.; Pugh, R.; Becker, D. \& Bacall, A. (2020). Identifying Critical 21st-Century Skills for Workplace Success: A Content Analysis of Job Advertisements. Educational Researcher, 49(2). https:/ / journals.sagepub.com/doi/10.3102/0013189X19890600 
Sandelowski, M. (2010). What's in a name? Qualitative Description revisited. Research in Nursing \& Health. 33, 77-84.

Santos Rego, M. A. (Edit.). (2020). La transferencia de conocimiento en educación. Un desafío estratégico. España: Narcea.

Van Der Westhuizen, D. W.; Pacheco, G. \& Webber, D.J. (2012). Culture, participative decisionmaking and job satisfaction. International Journal of Human Resource Management, 23(13), 2661-2679. http:// dx.doi.org/10.1080/09585192.2011.625967

Verea, N.; Naval, C. y Arbués, E. (2018). Innovación metodológica y responsabilidad social en la práctica docente universitaria. En Naval, C. y Arbués, E. (Eds.), Hacer la Universidad en el espacio social, 97-115. España: Eunsa.

Woodrufe, C. (1993). What is meant by a Competency? Leadership and Organization Development Journal, 14(1), 29-36. http:// dx.doi.org/10.1108/eb053651

Yuan, L.; Zhang, L. \& Tu, Y., (2018). When a leader is seen as too humble: A curvilinear mediation model linking leader humility to employee creative process engagement, Leadership \& Organization Development Journal, 39(4), 468-481. http://dx.doi. org/10.1108/LODJ-03-2017-0056 